\section{Strategies of proof}

\section{Graham Higman}

The Classification of Finite Simple

Groups. Vol. 1, Groups of

Noncharacteristic 2 Type.

By Daniel Gorenstein.

Plenum: 1983. Pp.487. \$59.50, £45.80.

THE completion of the classification of finite simple groups is one of the most exciting events of twentieth-century mathematics. The problem has intrinsic interest: finite symmetry groups are apt to crop up almost anywhere, and the simple groups are the blocks with which they are built. Thus questions about finite groups arise naturally. Knowing what the simple groups are does not necessarily trivialize them, but it does improve dramatically our chance of being able to answer them.

The list of finite simple groups is quite complicated. It has been known since the nineteenth century that the simple Lie algebras comprise four infinite series and five exceptional algebras. Each simple Lie algebra gives rise to a series of finite simple groups, one for each prime power. But, much as complex Lie groups can have more than one real form, sometimes there is more than one such series; and for more remotely analogous reasons, there are still further series when the prime power is $2^{n}$ or $3^{n}$. The members of this whole assemblage are called groups of Lie type. In addition, there are two further series, the cyclic groups of prime order and the alternating groups, and twenty-six odd men out, the so-called sporadic groups. The groups in the list are not only all formally different; they often have very different flavours. For instance, the groups of Lie type can be studied and are best studied by the powerful abstract and general methods of algebraic geometry, whereas the sporadic group have an awkward individuality as well as an intricate beauty, and never quite fulfil the promise they seem to give of something more general behind them.

Discovering and describing these simple groups is much less than half the battle. It has then to be proved that there are no others. Any such proof must somewhere reckon with each group in the list, and so can hardly be much less complicated than the list itself. Furthermore, the proof is put together from the contributions of a large number of mathematicians, working over several decades. In the early years, the later course of the argument could not be foreseen, so that papers written then are not always tailored to the needs of their successors. Key concepts, essential to the completion of the proof, often arose late in the day, and the significance of earlier work had to be revalued in the light of them. And, of course, there have been false trails, and seductive by-ways, and misunderstandings, and even mistakes. The proof is, indeed, there in the literature, but it can scarcely be said that it satisfies the requirement of accessibility to checking which is the mathematician's equivalent of the repeatability of experiments.

This is the situation to which Daniel Gorenstein has addressed himself in three books, of which the volume under review is the second, and which together seek to expand the whole proof, as it now is, in an accessible form. The first, entitled Finite Simple Groups, published in April 1982, described the groups in the list, gave an overview of the proof of completeness and developed the key concepts. The two remaining volumes put in the detail. It must be emphasized that, in spite of the length of the books, this is not detail for the sake of illustration or to clear up subsidiary questions; it is detail without which the proof would not be a proof. The method is determinedly historical, the expressed intention being to expound the original proof, with the improvements up to early 1982. Every step on the way is given the appropriate reference, so that the work constitutes a historical record as well as a mathematical exposition.

The proof of the classification theorem falls naturally into two parts. It has been clear since the pioneering work of Richard Brauer, and even more since the odd order paper of Walter Feit and John Thompson,

\section{Halley watching}

\section{David W. Hughes}

\section{International Halley Watch Amateur Observers' Manual for Scientific Comet Studies.}

By Stephen J. Edberg.

Enslow Publishers, Hillside, NJ / Sky Publishing, Cambridge, MA: 1983.

Pp.190. Pbk \$9.95.

OBSERVING Halley's comet in the winter of 1985 and spring of 1986 is going to be akin to ornithology. No serious bird-watcher would expect to leave his house and see a Wilson's Phalarope sitting on his gate post. To observe such an exotic species requires some effort: journeys to possible habitats, consideration of season, care over choice of observing instrument and so on.

Halley's comet at this apparition is going to be similarly hard to find and distinctly unstartling. Do not expect to walk outside and be amazed by a brilliant comet with a tail stretching from horizon to zenith. The geometry is unfavourable. When the comet is at its most active the Earth will be $1.5 \mathrm{AU}$ away on the other side of the Solar System. The coma magnitude will barely creep above the level visible with the naked eye for people in highly populated regions.

To see Halley's comet one must be well prepared and Edberg's Manual is an indispensable accessory. It has been written for the advanced amateur astronomer so it does not give guidance on basic observing that the key to the classification is the 2-local structure, the structure, that is, of the normalizers of subgroups of order a power of 2 . From this point of view, the groups of Lie type fall into two classes, according to whether the prime power involved is odd or even; and the distinction can be extended to other simple groups. This volume deals with the odd case; the second will deal with the even.

It cannot be doubted that Daniel Gorenstein was the right man to do this job. His personal contribution to the detailed work puts him among the top half-dozen workers in the field; but for generalship, for his early grasp of the wider strategies of the proof, he was quite outstanding. This book deserves the highest praise. It is not the last word on the subject, however; further rationalization of the existing proof is already on its way, and someone, perhaps, will come up with something quite new. And, no doubt, group theorists and users of group theory will go on using the classification theorem without knowing how to prove it. When the final volume appears in 1985 or 1986 , they will no longer have any excuse.

Graham Higman is Waynflete Professor of Pure Mathematics at the Mathematical Institute, University of Oxford.

techniques or methods of data reduction, but concentrates on explaining how the observer can generate meaningful scientific data. Part 1 introduces the International Halley Watch - the organization which will co-ordinate the observations and will eventually catalogue the data. Also stressed are the importance of visual observations of the coma and tail. This is one of the only techniques that can link present-day observations to those of many of the previous apparitions of the comet. The importance of dark adaptation, atmospheric transparency and sky brightness are emphasized. Photography (concentrating on the choice of emulsions, development processes, exposure times and lens focal length), astrometry (the accurate recording of the comet's position), spectroscopy, photoelectric photometry and observations of the two associated meteor streams are also covered.

Part 2 consists of an ephemeris (which, among other things, lists right ascension, declination, magnitude, heliocentric and geocentric distance at daily intervals between June 1985 and May 1987), a set of 19 star charts showing the movement of the comet against the sky background, and five figures showing the elevation and azimuth of the comet for observers at latitudes $40^{\circ} \mathrm{N}, 30^{\circ} \mathrm{N}, 20^{\circ} \mathrm{N}, 20^{\circ} \mathrm{S}$ and $30^{\circ} \mathrm{S}$.

No amateur or professional astronomer who has the vaguest interest in comets should be without this book.

David $W$. Hughes is a Lecturer in Physics and Astronomy at the University of Sheffield. 